\title{
A wind effect of neutron component of cosmic rays at Antarctic station Mirny
}

\author{
Kobelev Pavel \\ IZMIRAN. Kalushskoe Ave., 4, Moscow, Troitsk, Russia, 142190; E-mail: kosmos061986@yandex.ru
}

Pustinik Lev ${ }^{1}$

Israel Cosmic Ray and Space Weather Center Institute of Advanced Study of Tel Aviv University, P.O. Box 39040,Tel Aviv,69978, ISRAEL; E-mail: levpust@post.yau.ac.il

\section{Belov Anatoly}

IZMIRAN. Kalushskoe Ave., 4, Moscow, Troitsk, Russia, 142190; E-mail: abelov@izmiran.ru

\section{Eroshenko Eugeniya}

IZMIRAN. Kalushskoe Ave., 4, Moscow, Troitsk,Russia, 142190;E-mail: erosh@izmiran.ru

\section{Gushchina Raya}

IZMIRAN. Kalushskoe Ave., 4, Moscow, Troitsk, Russia, 142190;E-mail:rgus@izmiran.ru

\section{Smirnov Dmitry}

IZMIRAN. Kalushskoe Ave., 4, Moscow, Troitsk, Russia, 142190; E-mail : smirnovdm@yandex.ru

\section{Yanke Victor}

IZMIRAN. Kalushskoe Ave., 4, Moscow, Troitsk, Russia, 142190; National Research Nuclear University MEPHI (Moscow Engineering Physics Institute), Kashirskoe highway, 31, Moscow, Russia, 115409; E-mail: vanke@izmiran.ru

\begin{abstract}
Estimation of barometric coefficient for neutron component of cosmic rays was performed for Antarctic station Mirny taking into account effect of dynamic pressure caused by wind in the atmosphere. Hourly data of continue monitoring of neutron component and data of the local meteo station have been used for the period 2007-2014. Wind velocity at the observatory Mirny reaches $20-40 \mathrm{~m} / \mathrm{s}$ in winter that corresponds to dynamic pressure of 5-6 mb and leads to the error of 5\% in variations of neutron component because of dynamic effect in the atmosphere. The results are interesting for high latitude and high mountain detectors, where effect Bernulli may be significant.
\end{abstract}

The 34th International Cosmic Ray Conference

30 July- 6 August, 2015

The Hague, The Netherlands

\footnotetext{
${ }^{1}$ Speaker
} 


\section{Introduction}

The most precisions and stable detector which more than half a century provides data for investigation of the primary cosmic ray variations is a neutron monitor. The statistical accuracy of the standard neutron supermonitor $18 \mathrm{~nm} 64$ at sea level is about (in the hourly averaging) $0.15 \%$, so it is necessary to errors from other possible sources to be lower then this. Primarily, such possible sources include the errors of exclusion a barometric effect from observed data. Typical accuracy of modern pressure sensors is $0.2 \mathrm{mb}$, which gives us the required accuracy of amendments $\approx 0.15 \%$. But there is another source of errors, more difficult for consideration. Barometric effect which primarily is caused by neutron absorption in the atmosphere, is determined by the amount of matter above the detector, i.e. by static pressure. Applicable pressure sensor measures the total pressure as the sum of the static and dynamic ones. The objective of this work is the experimental determination of the contribution of the dynamic pressure and the application of necessary corrections to the observed data.

Dynamic pressure is caused by wind flow and equal to the kinetic energy of the unit volume of matter $P_{D}=1 / 2 \rho V^{2}$, where $\rho$ - is the density of the air, $V$ - is a flow speed.

However, only a part of the kinetic energy is $C_{x} P_{D}$ converted into potential energy and impacts on an obstacle and on the visible data of pressure sensor. The proportionality coefficient (an aerodynamic coefficient) depends on the geometry of the obstacles and the Reynolds number, i.e. the level of turbulence. The effect of wind for the individual events was investigated in [1-6], but a detailed analysis was not performed.

\section{Experimental data}

Regular monitoring of space and meteorological parameters at Mirny station is carried out since 2007. The following data have been used in this work: the values of the counting rate of the neutron monitor 12nm64, pressure sensor RSB-1M measurements (this sensor located in the same room with the detector $12 \mathrm{~nm} 64$ ), pressure and wind velocity vector according to the weather station MILOS-500 (at a distance of 900 meters from the first pressure sensor).

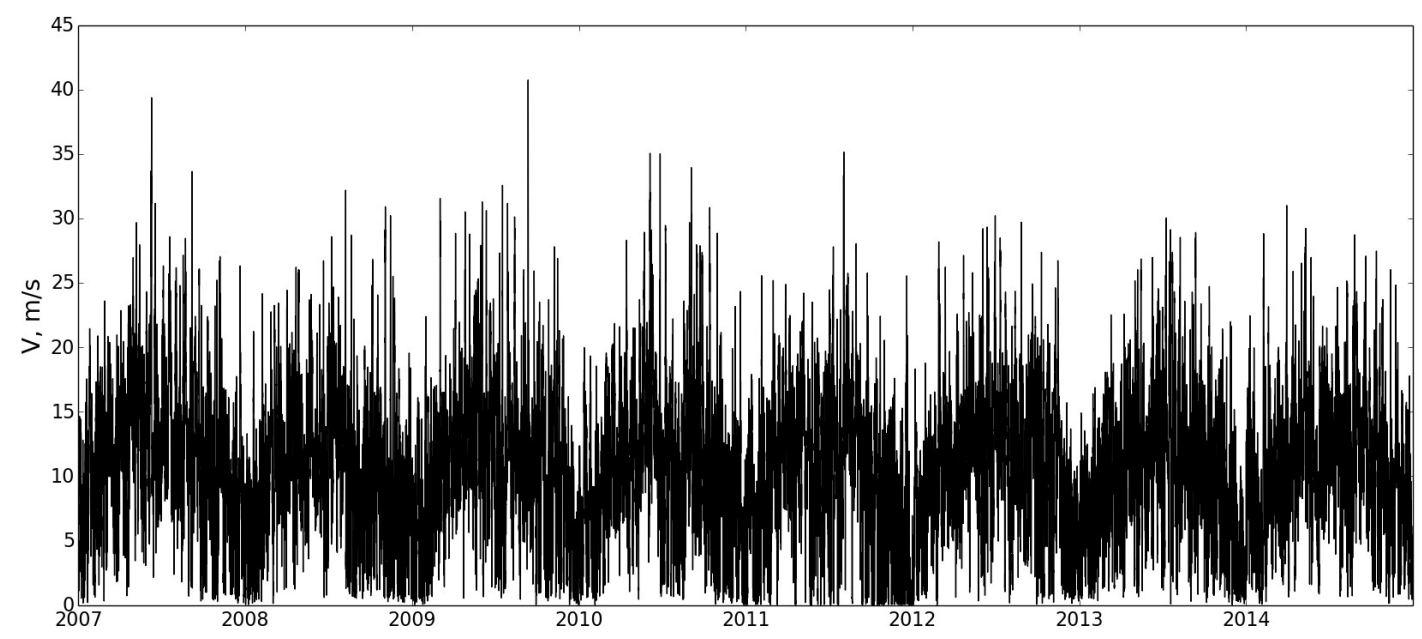

Figure 1. Observed at observatory Mirny wind velocity during 2007-2015. 
Actually analysis was based on the hourly data use classic method [7]. To estimate the turbulence also used atmosferic pressure and wind speed at hour resolution. To estimate the turbulence also used atmospheric pressure and wind speed at minute resolution. All data is organized in a database "Mirny" [8], which contains two tables of identical structure for the data

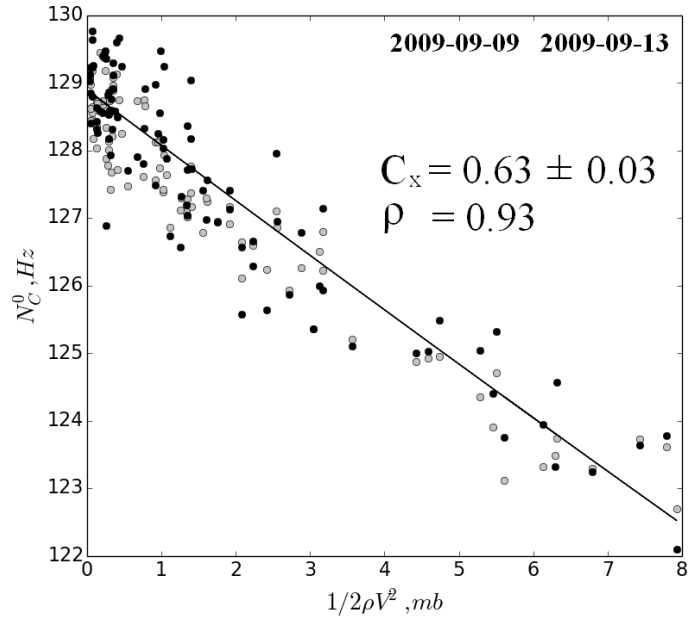

Figure 2. Correlation dependence of uncorrected and corrected for the primary variations counting rate (grey and black points correspondingly) on dynamic pressure of wind; curve NMK is approximation for corrected data.

at hour and minute resolutions.

On the Antarctic stations, including station Mirny, due to the nature of the relief "run off" winds are observed. The strength of the "run off" (stock) wind is proportional to the steepness of the slope and reaches the highest values in the coastal areas with high slope towards the sea. The maximum force stock winds reach at the Antarctic winter from April to November, they blow almost continuously day and night. Figure 1 shows the observed wind speed over 2009-2014 in minute resolution.

\section{Method}

Barometric effect is easily eliminated by the law of radiation absorption in the atmosphere according to the deviation of the measured atmospheric pressure (assuming that it is the static pressure $\mathrm{P}_{\mathrm{S}}$ ) from the standard $\mathrm{P}_{0}$ for the observation point

$$
N_{C}=N_{U} \cdot \exp \left[-\beta\left(P_{0}-P_{S}\right)\right],
$$

where $\beta=1 / \mu$, the so-called barometric coefficient and $\mu$ is free path of the particles in the atmosphere, $N_{U}$ - the measured detector counting rate, $N_{C}$ - the detector counting rate, reduced to the standard level $P_{0}$.

Because measured by the sensors barometric pressure $P$ is the sum of the static and dynamic pressure of the atmosphere at this point, the static pressure $P$ equals the difference between the measured and the dynamic pressure, i.e. $P-C_{x} P_{D}$.

Corrected for the barometric effect (static pressure) detector counting rate $N_{C}$ can be represented as:

$$
N_{C}=N_{U} \cdot e^{-\beta\left[P_{0}-\left(P-C_{x} P_{D}\right)\right]}=\underbrace{N_{U} \cdot \exp \left(-\beta\left(P_{0}-P\right)\right)}_{N_{C}^{0}} \cdot \exp \left(-\beta C_{x} P_{D}\right),
$$

where $N_{U}$ - registered count rate detector at the moment and $\mathrm{P} 0$ - the average pressure over some time interval. Barometric coefficient $\beta>0$ ( $\beta$ for Mirny station is $0.73 \% / \mathrm{mb}, P_{0}=980$ $\mathrm{mb}$ ) is defined in a calm and windless period. Logarithming of equation (2), we got the linear equation:

$$
\ln N_{C}^{0}=\ln N_{C}+\beta C_{x} p_{D} \text { or } y=a+c x,
$$

where $y=\ln N_{C}^{0}, a=\ln N_{C}, x=\beta P_{D}$, i.e. linear regressive equation with respect to $a$ and $c$. 
Next, data should be corrected on the primary variations. To solve the problem of estimating the barometric effect, measured by detector counting rate $N_{U}$ should be freed from the primary variations. This can be done, if replace $N_{U}$ by $N_{U} /(1+v)$, where $v$ - the primary variation for this point of observation. Then equation (2) can be written as:

$$
N_{C}=\underbrace{N_{U} /(1+v) \cdot \exp \left(-\beta\left(P_{0}-P\right)\right)}_{N_{C}^{0}} \cdot \exp \left(-\beta \cdot C_{x} P_{D}\right),
$$

In approach of zero harmonic, the primary variations can be eliminated according to the data of the base, near-located station $\mathrm{S}$ by follows. Let's write variations for the two detectors as:

$$
v=a_{10} \cdot C_{0} \text { and } v^{S}=a_{10} \cdot C_{0}^{S}
$$

Eliminating the unknown amplitude of zero harmonic $a_{10}$, we can estimate the expected variation of the test detector by means the observed variations in the detector $\mathrm{S}$

$$
v=v^{S} \frac{C_{0}}{C_{0}^{S}}
$$

Equation (5) accounts the difference in parameter of the stations (height, stiffness geomagnetic cutoff) and, in principle, allows you to involve any of the stations as a base referenced.

\section{Discussion of the results}

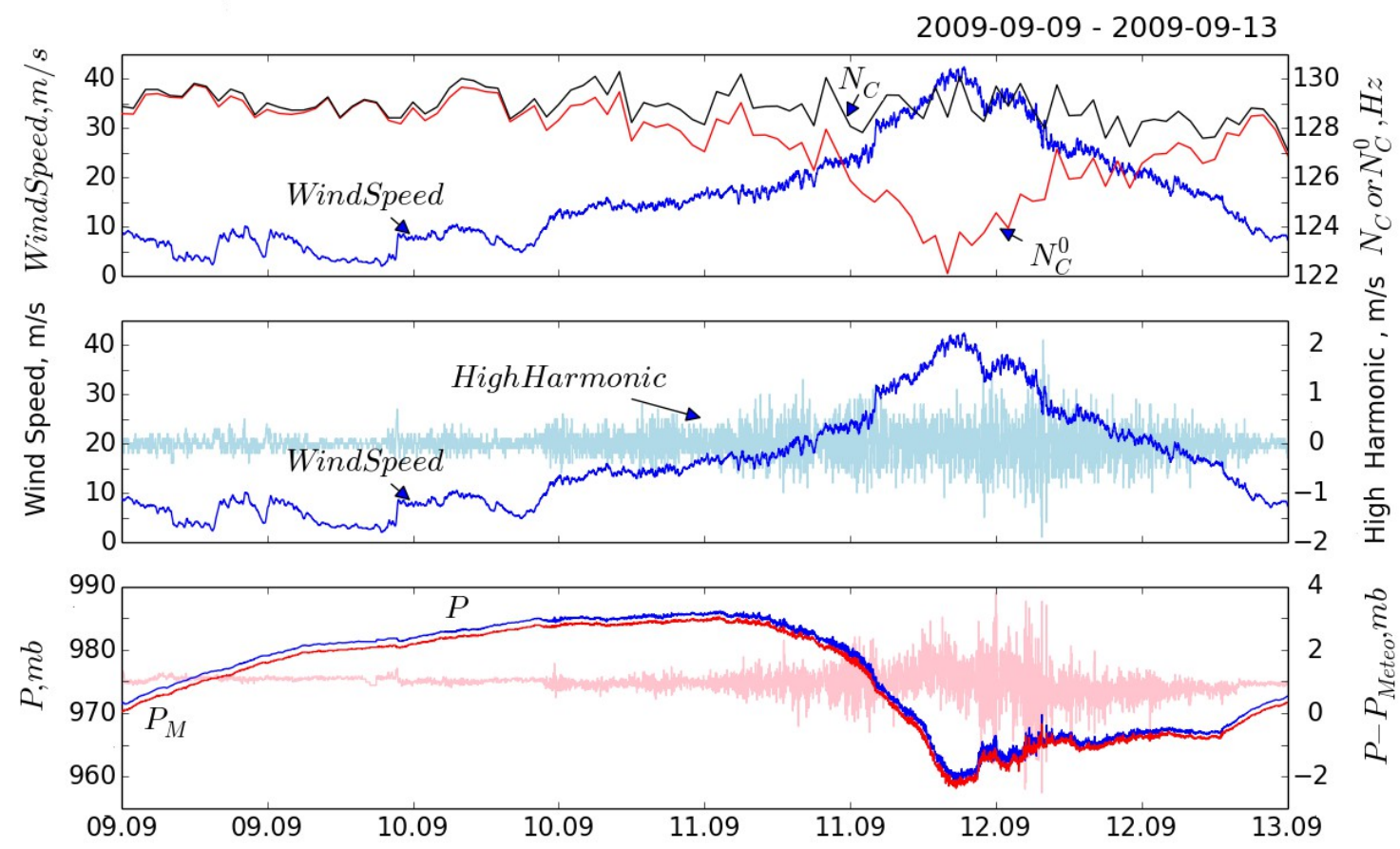

Figure 3. Temporary dependences of corrected on barometric effect counting rate with taking into account the effect of wind and without it $\left(N_{C}^{0}\right.$ и $N_{C}$ ), and also wind speed (upper panel). The time dependence of the values of the wind speed in minute resolution and a filter adjacent differences (middle panel). Indications two pressure sensors $\mathrm{P}$ and $\mathrm{Pm}$ and their difference (lower panel).

For the analysis we have selected about a dozen of events, when the observed wind speed exceeded $30 \mathrm{~m} / \mathrm{s}$. For example, we show a record event of September 2009, when the maximum 
wind speed reached $42 \mathrm{~m} / \mathrm{s}$. Figure 2 shows the correlation between the counting rate and the calculated dynamic pressure $P_{D}$. Counting rate (gray circles) by above described procedure (formula 4) was corrected for primary variations (black circles). Correlation analysis for this event leads to aerodynamic coefficient $C_{x}=0.63 \pm 0.03$, (the correlation coefficient is 0.93 ). Of all considered the events this correlation coefficient turned out to be the best.

Strictly speaking, the aerodynamic coefficient depends on Reynolds number, which is proportional to wind speed. But in a limited range of speeds, this dependence can be neglected if the Reynolds number is out of the critical region of crisis resistances $[9,10]$.

Details of the analysis are presented in Figure3. At the top of the figure the time dependences of corrected for the barometric effect counting rates without and with taking into account the wind effect, and also time dependence for wind speed are shown. It is clearly visible

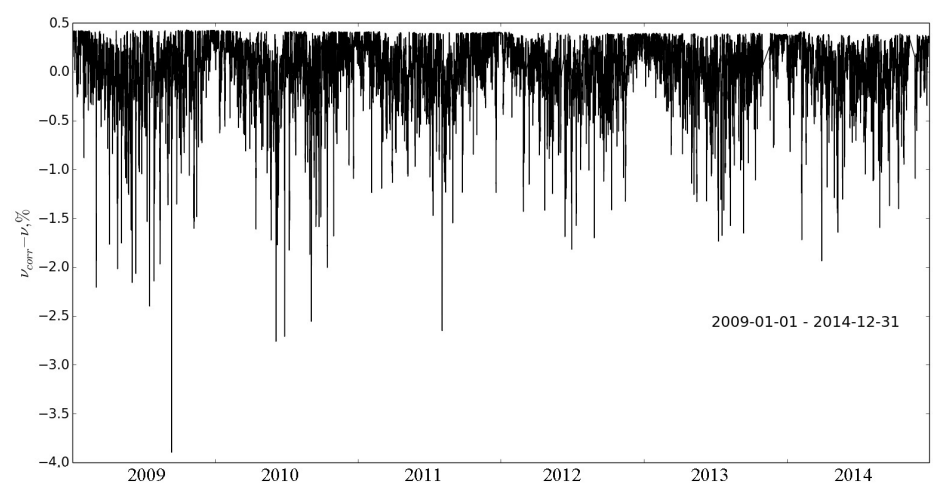

Figure 4. Correction for the dynamic effect the observed variations of cosmic rays during the period 2009-2014. a good anti-correlation between counting rate $N_{C}^{0}$ and wind speed, which is completely removed after the correction for the dynamic effect in accordance with the expression (4). In the middle panel of the figure the time dependence of the wind speed (in minute resolution) and the filter adjacent differences are shown. It can be seen, that at wind speeds

exceeding $15 \mathrm{~m} / \mathrm{s}$ a turbulence of the flux increases; this fact should be taken into account under further investigations. In the lower portion of Figure 3 readings of two pressure sensors (placed

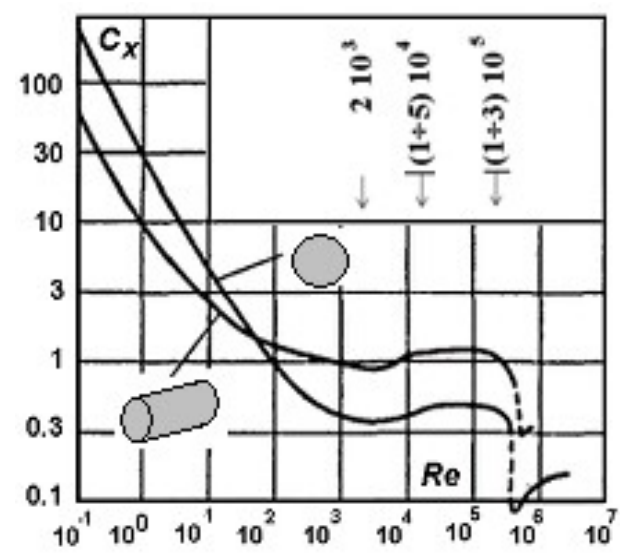

Figure 5. Зависимость аэродинамического коэффициента [9] от числа Рейнольдса для шара и цилиндра. inside and outside of the building) and their difference (minute resolution) are shown: according to these results, readings of different placed pressure sensors are identical in lowwind speed periods and have fluctuations at high speeds, which confirm the flow turbulence. Turbulence that observed with the free pressure gauge measurements $\mathrm{P}_{\mathrm{M}}$, is smaller than in the case of the pressure sensor $\mathrm{P}$ inside the building, due to the condition of airflow building.

This raises the question on dependence of the aerodynamic coefficient on the wind speed, i.e., on the Reynolds number.

The presence of a significant dissipation of energy throughout the volume of the turbulent wake, as well as the formation of the interface

in the separation of the boundary layer, lead to the fact that the bodies, under airflow of which the flow boundary layer separation occurs, providing a high resistance to the oncoming flow. At 
sufficiently high Reynolds numbers, at which, however, the boundary layer remains laminar up to separation point, the coefficient of resistance is not dependent on Re. However, when the Reynolds number reach the values at which the boundary layer is turbulized to separation point of the laminar boundary layer, a separation point of the boundary layer moves downstream; herewith turbulent layer narrows considerably, and the resistance of the body is reduced by several times. This phenomenon is called resistance crisis, and appears because of fact that the transfer of momentum in the boundary layer sharply increases. From the latter it follows, that the entrainment of substance within the boundary layer by external flow is significantly enhanced, and the liquid particles move in the boundary layer at the direction of the pressure rise further, than in laminar boundary layer case.

You can put the question about dependence of aerodynamic coefficient from Reynolds numbers, obtained for different values of wind speed for the test period. The Reynolds number $\operatorname{Re}=V \cdot L / v$ for observed at the observatory conditions varies from $3.410^{7}$ to $1.510^{8}$ at changing of wind speed V of $10 \mathrm{~m} / \mathrm{s}$ to $45 \mathrm{~m} / \mathrm{s}$ and the characteristic size $\mathrm{L}=40 \mathrm{~m}$, i.e. Reynolds number, even for low values of wind speed is higher than the value of the Reynolds number Re $=310^{5}$ at crisis resistance. For ground level conditions at $\mathrm{t}=-20^{\circ} \mathrm{C}$ and the air density $\rho$ $=1.393 \mathrm{~K} 2 / \mathrm{M}^{3}$, the following values for the dynamic $\left(\mu=1.6310^{-5} \mathrm{~N} \mathrm{~s} / \mathrm{m}^{2}\right)$ and kinematic ( $\left.v=\mu / \rho=1.1710^{-5} \mathrm{M}^{2} / \mathrm{s}\right)$ viscosity of air have been used.

Aerodynamic coefficients $C_{x}$ obtained allow us to consider the dynamic effects for the entire period of observation, as shown in Figure 5. Because of accuracy of the observed variations is tenths of a percent, the possible error due to a dynamic effect, as seen in the figure, reaches several percents under strong winds.

\section{Conclusions}

It is shown that at Mirny station, where wind speeds often reach high values, the absolute error in the determination of variations may reach $2-4 \%$, thus, to obtain precise data we should always recalculate barometric effect with taking into account the dynamic effect of the wind. To identify the depending the aerodynamic coefficient on the Reynolds number we need to consider larger number of events. In addition, it is very important to consider the dynamic effects at other polar detectors, primarily neutron monitor Mawson, where the greatest regular winds are observed. For example, the annual average wind speed at the station Mawson is $21 \mathrm{~m} / \mathrm{s}$, and at station Mirny is 11 $\mathrm{m} / \mathrm{s}$ [11]. It is also important to involve data of mountain detectors, because there are very different conditions of flow around obstacles for them.

\section{Acknowledgements}

This work is partly supported by Program № 10 BR of the Presidium RAS "The fundamental properties of matter and Astrophysics”, Program № 22 BR of the Presidium RAS "Fundamental processes of research and development of the Solar System". The authors are thankful to collaborators of the world wide network of CR stations providing data of continue monitoring of neutron component: http://cr0.izmiran.ru/ThankYou. We acknowledge also the 
NMDB database (www.nmdb.eu), founded under the European Union's FP7 program (contract no. 213007) for providing data from NM worldwide network.

\section{References}

[1] Buticofer R., Flugiker E.O., "Pressure correction of GLE measurements in turbulent winds", Proc. 26th ICRC, Salt Like City, 6, 395-398, 1999.

[2] Dorman L.I., Villoresi G., Iucci N., Parisi M., Ptitsyna N.G., 1999. “Cosmic ray survey to Antarctica and coupling functions for neutron component in solar minimum (1996-1997), 3. Geomagnetic effects and coupling functions", Proc. of 26-th Intern. Cosmic Ray Conference, Salt Lake City, V7, p. 382-385.

[3] Dubinsky J., Chaloupka P., Kowalsky T., Mat.-fys. casop., 10(1), 57, 1960.

[4] Kawasaki S., "On the anomalous barometric coefficient of cosmic-ray neutron monitor at Mt. Norikura", Sci. Rap. of Inst. Phys. Chem. Res., 66(2),25-32, 1972.

[5] Lockwood J.A., Calawa A.R., "On the barometric pressuce coefficient for cosmic ray neutrons", J.Atmos.and Terr.Phys., 11,23, 1961.

[6] Dorman, Lev I., Cosmic Rays in the Earth's Atmosphere and Underground, Astrophysics and Space Science Library, Vol. 303, Kluwer Academic Publishers, Dordrecht, pp.855, 2004.

[7] Kobelev P., Belov.A, Mavromichalaki.E, Gerontidou M., Yanke V., "Variations of Barometric Coefficients of the Neutron Component in the 22-23 Cycles of Solar Activity", Proc. 32nd ICRC, 2011.

[8] DB Mirny. ftp://crsb.izmiran.ru/Mirny, 2007.

[9] Monin A., Yaglom A. "Statistic Hydromechanic". M.: "Nauka", 640 c., 1965. http://sci.sernam.ru/book_s gidro.php?id=11.

[10] Shakina N. Lectures by dynamic metheorology. - M.: Triada ltd.,160 c. , 2013, http://method.meteorf.ru/publ/books/lectures/lectures.pdf.

[11] WorldWeather http://www.tutiempo.net/en/Climate or http://rp5.ru,2015. 